\title{
Clinical Practices and Outcomes on Chemotherapy-Induced Nausea and Vomiting Management in South Korea: Comparison with Asia-Pacific Data of the Pan Australasian Chemotherapy Induced Emesis Burden of IIIness Study
}

\author{
Myung Ah Lee, MD, PhD \\ Eun Kyung Cho, MD, PhD² \\ Sung Yong $\mathbf{O h}, \mathrm{MD}, \mathrm{PhD}^{3}$ \\ Joong Bae Ahn, MD, PhD ${ }^{4}$ \\ Ji Yun Lee, MD, PhD ${ }^{5}$ \\ Thomas Burke, $\mathrm{PhD}^{5}$ \\ Hun Jung, MD, PhD ${ }^{5}$ \\ Jong Gwang Kim, MD, $\mathrm{PhD}^{6}$
}

\section{Purpose}

This study reported patient outcomes of chemotherapy-induced nausea and vomiting (CINV) prophylaxis for highly emetogenic chemotherapy (HEC) and moderately emetogenic chemotherapy (MEC) regimens and evaluated its adherence to acute-phase CINV prophylaxis in the Korean population subset of the Pan Australasian Chemotherapy Induced Emesis burden of illness (PrACTICE) study.

\section{Materials and Methods}

This subgroup analysis evaluated 158 Korean patients receiving HEC or MEC and compared the data (wherever possible) with that of 648 patients from the Asia-Pacific (AP) region. Study endpoints included evaluation of primary CINV prophylaxis and adherence to acutephase CINV prophylaxis in cycle 1 (American Society of Clinical Oncology [ASCO] Quality Oncology Practice Initiative [QOPI]).

\section{Results}

In South Korea and the AP, a 5-hydroxytryptamine-3 receptor antagonist $\left(5 \mathrm{HT}_{3}-\mathrm{RA}\right)$ prophylaxis for the acute phase was administered to $79 / 80$ patients (98.8\%) for HEC and 70/71 patients (98.6\%) for MEC regimens (QOPI-1). Triple regimen (corticosteroid-5HT - RA-neurokinin 1-RA) was initiated in $46 / 80$ patients (57.5\%) for prophylaxis of acute CINV in cycle 1 of $\mathrm{HEC}$ (QOPI-3). Double regimen (corticosteroid-5HT $-\mathrm{RA}$, with or within $\mathrm{NK}_{1}-\mathrm{RA}$ ) was initiated in 61/71 patients (83.1\%) for control of acute CINV in cycle 1 of MEC a(QOPI-2).

\section{Conclusion}

Active management of CINV is necessary in cycle 1 of HEC in South Korea, despite higher rates than the AP region. Adherence to the international guidelines for CINV prophylaxis requires attention in the acute phase in cycle 1 of the HEC regimen.

\footnotetext{
Correspondence: Jong Gwang Kim, MD, PhD Department of Oncology/Hematology, Kyungpook National University Medical Center, Kyungpook National University School of Medicine, 807 Hoguk-ro, Buk-gu, Daegu 41404, Korea

Tel: $82-53-200-2623$

Fax: 82-53-200-2019

E-mail: jkk21c@knu.ac.kr

Key words

Nausea, Vomiting, Drug therapy, Antiemetics 


\section{Introduction}

Chemotherapy-induced nausea and vomiting (CINV) significantly impairs quality of life and adherence to planned chemotherapy regimens in cancer patients. Despite the availability of newer antiemetic agents, reducing the incidence of CINV remains a challenge, particularly in the case of nausea and delayed CINV (occurring $>24$ hours postchemotherapy) $[1,2]$. Although the incidence varies with the chemotherapy regimen, choice of antiemetic, adherence to antiemetic guidelines, and patient characteristics, ethnic differences, and genetic polymorphisms are also involved as they influence the metabolism of antiemetic agents [1,3-5].

Several guidelines recommend prophylactic antiemetic regimens for anticipatory nausea and vomiting and for the acute and delayed phases of treatment, based on choice of chemotherapeutic agents. As anticipatory nausea and vomiting shows a poor response to treatment, antiemetic guidelines recommend prevention with optimal first-line antiemetic prophylaxis for acute and delayed CINV [6-9]. However, research shows that adherence to guidelines is low, and antiemetics are typically under prescribed in patients receiving highly emetogenic chemotherapy (HEC) or moderately emetogenic chemotherapy (MEC) regimens, with wide variations in dosage leading to suboptimal control of CINV $[1,10$ 13].

The Pan Australasian Chemotherapy Induced Emesis burden of illness (PrACTICE) study evaluated the burden of CINV among patients receiving HEC or MEC in six countries across the Asia-Pacific (AP) region [14-18]. Data pertaining to the incidence of CINV in various chemotherapy cycles $[14,16]$, the pattern of CINV prophylaxis in practice [15], predictors of anticipatory CINV [17], and the influence of CINV on modifications made to earlier cycles of chemotherapy regimens [16] have been previously published. The results of these studies demonstrated that CINV in prior cycles was a strong and consistent predictor of CINV in subsequent cycles, and the incidence of chemotherapy regimen modification because of CINV was low in individual cycles [16], thus highlighting the importance of preventing CINV in cycle 1 to reduce anticipatory nausea and vomiting in subsequent cycles [17]. Differences in the prevalence of qualityof-care indicators, adherence to guidelines, and prescribing patterns of CINV prophylaxis for HEC and MEC within and across countries [15] were also noted. The 5-hydroxytryptamine- 3 receptor antagonists $\left(5 \mathrm{HT}_{3}\right.$ - $\left.\mathrm{RAs}\right)$ were the most consistently prescribed antiemetics in the AP region; prescriptions for other antiemetic therapies were variable [15]. The highest prescribing behavior and use of rescue medications were noted in Australia and Singapore, whereas the lowest use was noted in India, South Korea, and Taiwan. In addi- tion, country-specific differences can provide important information for designing studies and implementing country-specific guidelines [14]. As CINV remains a substantial problem, country-specific information could also improve outcomes for patients undergoing chemotherapy [14]. These differences suggest that clinical guidelines must be adapted based on country- and area-specific healthcare systems in addition to drug availability, reimbursement policies, and clinical practices. Although studies on CINV have been conducted in the AP region [19-22], differences in study design and location prevent broad generalizations. PrACTICE is the only study with a common study design evaluating the burden of CINV in six countries of the AP region [18].

The current study reports on the subgroup analysis of patient outcomes, including CINV prophylaxis in cycle 1 of HEC and MEC and evaluated the adherence of this acutephase CINV prophylaxis in cycle 1 according to the criteria of measures in the American Society of Clinical Oncology (ASCO) Quality Oncology Practice Initiative (QOPI) in the Korean population subset of the PrACTICE study.

\section{Materials and Methods}

\section{Study design}

PrACTICE, a multicountry, multisite, prospective, observational study evaluated the burden of emesis in adult patients initiating HEC or MEC for cancer treatment at 31 sites in six countries (Australia, China, India, Singapore, South Korea, and Taiwan) across the AP region. The detailed study design has been previously published [18]. The study was conducted in accordance with the Declaration of Helsinki and local regulations. Patients provided written informed consent before enrollment, and the study was approved by the institutional review board of each institute.

In the subgroup analysis, we evaluated patient outcomes pertaining to CINV among the 158 Korean patients enrolled. Patients were categorized according to HEC and MEC groups. Wherever possible, the Korean data were compared to the data of 648 patients enrolled across the AP region. The primary endpoint was the prescription pattern for cycle 1 of CINV prophylaxis in South Korea. Secondary endpoints included adherence to acute-phase CINV prophylaxis in cycle 1 to the criteria of measures in the ASCO QOPI and the primary source of consulting guidelines for CINV prophylaxis. 


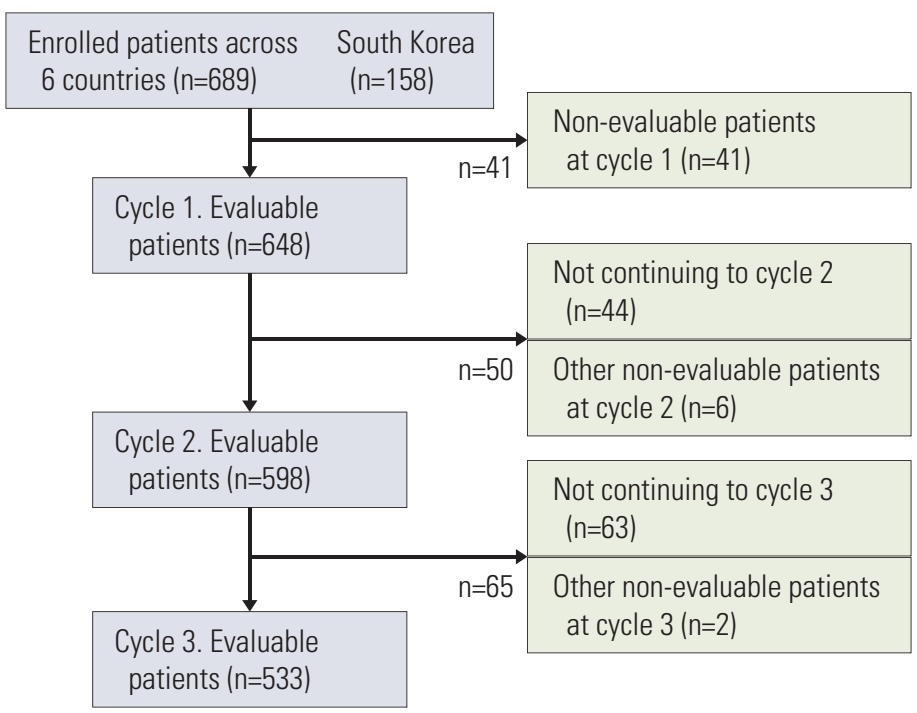

Fig. 1. Distribution of enrolled patients in PrACTICE (CONSORT flow diagram). PrACTICE, Pan Australasian Chemotherapy Induced Emesis burden of illness.

\section{Study population}

Male and female patients aged $\geq 18$ years and naïve to HEC and MEC [18] were considered eligible. They were to receive at least two cycles of cancer chemotherapy with intravenous administration of at least one of the following commonly used single-day HEC or MEC agents: cisplatin, cyclophosphamide, dacarbazine, oxaliplatin, carboplatin, doxorubicin, epirubicin, or irinotecan. Chemotherapy regimens were categorized as HEC or MEC based on the emetogenic potential of the single agent with the highest emetic risk in a regimen [22]. Chemotherapy agents with low emetogenic potential were permitted if the patient had received at least one of the HEC or MEC agents. Patients not capable of taking oral medications, scheduled multiday chemotherapy, concurrent radiotherapy, current brain metastases, or vomiting in the 24 hours before cycle 1 were excluded from the study. Additional details have been described in a previous publication [18].

\section{Assessments and outcomes}

The primary investigator at each site completed a one-time survey regarding adherence to antiemetic guidelines before enrolling patients. Enrolled patients answered a questionnaire-based survey (in the form of a diary) before receiving chemotherapy (on day 1) and on days 2 and 6 of each chemotherapy cycle. These included questions regarding vomiting, nausea, and anticipation of nausea, and anxiety during the 24 hours before initiating chemotherapy for each cycle. Similarly, patients completed the Multinational Association of Supportive Care in Cancer (MASCC) antiemesis tool (MAT) (a validated tool for measuring the incidence and severity of nausea and vomiting after chemotherapy [23]) on days 2 and 6 of each cycle. Questions included in the diary for cycles 1 through 3 are outlined in a previous publication [18]. Patients who completed the questionnaire in the diary for cycle 1 continued to receive study therapy, maximum of three cycles, for approximately 3-4 weeks (Fig. 1). Patients also completed an antiemetic medication questionnaire regarding use of medications for CINV prophylaxis or as rescue therapy for treatment of established nausea or vomiting in the 24 hours after chemotherapy (acute phase) or from 24 hours to 5 days after chemotherapy (delayed phase) on days 2 and 6 of each cycle. Details of chemotherapy, including date, time, setting (inpatient or outpatient), route of administration, type of chemotherapy, and acute-phase CINV prophylaxis were recorded on day 1 of each cycle. Similarly, delayed-phase CINV prophylaxis prescribed for days 2-5 was recorded. Thereafter, anonymized electronic case report forms containing patients' responses to the study questionnaires and their complete medical record data were generated. The study did not influence treatment decisions or any site-specific investigations.

The primary antiemetics were classified according to three categories based on the World Health Organization Drug Dictionary, September 1, 2010, ver. 3 [24]. These included corticosteroids, neurokinin-1 receptor antagonists ( $\mathrm{NK}_{1}-\mathrm{RAs}$; aprepitant, and fosaprepitant), and $5 \mathrm{HT}_{3}-\mathrm{RAs}$ or setrons (granisetron, ondansetron, palonosetron, ramosetron, and 
tropisetron). Categories of secondary antiemetics included antihistamines, benzodiazepines, histamine- 2 receptor antagonists, propulsives (e.g., domperidone and metoclopramide), proton-pump inhibitors (e.g., lansoprazole, omeprazole, and pantoprazole), and others (e.g., haloperidol, prochlorperazine, and pyridoxine). Due to differences in reimbursement and/or regulatory status across countries in the AP region, use of $\mathrm{NK}_{1}-\mathrm{RA}$ (aprepitant) differed by country. Aprepitant prescribed for HEC and for secondary prevention in MEC is eligible for reimbursement in Australia and Korea. Aprepitant had regulatory approval in Taiwan, Singapore, and India; however, it was not eligible for reimbursement during the study period. Aprepitant did not have regulatory approval in China during the study.

Quality-of-care indicators were based on the ASCO QOPI [6] and categorized as follows according to adherence to the criteria of measures in the ASCO QOPI Symptom/Toxicity Management-Chemotherapy-Related Module for acutephase CINV prophylaxis.

1) QOPI-1: $5 \mathrm{HT}_{3}-\mathrm{RA}$ prophylaxis prescribed or administered with HEC and MEC (corresponds to QOPI Symptom Module \#26)

2) QOPI-2: corticosteroid and $5 \mathrm{HT}_{3}$-RA prophylaxis prescribed or administered with HEC and MEC (corresponds to QOPI Symptom Module \#27)

3) QOPI-3: aprepitant prescribed with HEC (corresponds to QOPI Symptom Module \#28)

4) QOPI-4: antiemetic therapies appropriately prescribed for HEC and MEC; thus, for HEC, yes to QOPI-2 and QOPI-3 and for MEC, yes to QOPI-2 (corresponds to QOPI Symptom Module \#29)

Patients were categorized based on adherence of their acute-phase CINV prophylaxis in cycle 1 according to the criteria of measures in the ASCO QOPI.

\section{Statistical considerations}

Study outcomes, including CINV incidence and treatment patterns (chemotherapy or CINV prophylaxis), were descriptively analyzed by phase (overall, acute, or delayed), by cycle (as appropriate), within and across countries, and by level of chemotherapy emetogenicity (HEC vs. MEC). For analysis, patients were assigned to the following mutually exclusive chemotherapy regimens: HEC, wherein the treatment parameters were cisplatin-based, intravenous cyclophosphamide-based $\left(\geq 1,500 \mathrm{mg} / \mathrm{m}^{2}\right)$, dacarbazine-based, and women treated with anthracycline plus cyclophosphamide; and MEC, wherein the treatment parameters were carboplatin, cyclophosphamide $<1,500 \mathrm{mg} / \mathrm{m}^{2}$, doxorubicin (nonpegylated and nonliposomal), epirubicin, irinotecan, and oxaliplatin. Differences in achievement of complete response between countries were assessed using Pearson's chi-square test. Statistical significance was assessed at a two-sided 0.05 level. Unless otherwise stated, all confidence intervals (CIs) were at the 95\% level and two-sided. For the summary of percentages with limited sample size, including countrylevel summaries, the Clopper-Pearson exact CI for binomial distribution was used. Further details are available in a previous publication [18]. Statistical analyses were used for the summary of percentages with limited sample size, including country-level summaries" and performed using SAS ver. 9.2 (SAS Institute Inc., Cary, NC). The primary goal of the study was to provide South Korea-specific data on CINV outcomes that could potentially be used to improve the quality of antiemetic prescribing in the region. The sample size calculation was previously described [18].

The ASCO QOPI measures are reported as counts and percentages for patients meeting each of the four criteria for the acute phase, summarized by cycle and country. Analysis of the ASCO QOPI adherence is described in detail in a previous publication [15].

\section{Results}

The PrACTICE study enrolled 689 patients across six countries in the AP region with 158 patients in South Korea, with 648 patients evaluable in cycle 1. Enrolled patient distribution is shown in Fig. 1. The subgroup analysis included 151 Korean patients who received cycle 1 of chemotherapy. Evaluable patients in South Korea initiated on HEC included 80 patients in cycle 1, 74 in cycle 2, and 68 in cycle 3 . Similarly, evaluable patients initiated on MEC included 71 patients in cycle 1, 63 in cycle 2 , and 56 in cycle 3 . The distribution of evaluable patients receiving cycles 1,2 , and 3 of HEC and MEC across countries is shown in Table 1.

Baseline patient characteristics are described in detail for each cohort, overall and by country, in a previous publication [14]. The Korean patient distribution across the HEC and MEC regimens based on type of cancer is shown along with corresponding AP data in Table 2.

In the HEC group, cisplatin was the most commonly used agent in $75 \%$, and doxorubin was the next most commonly used in $26.3 \%$. In the MEC group, most patients were treated with oxaliplatin (93\%). 5-Fluorouracil or capecitabine was also commonly used in $28.2 \%$ and $29.6 \%$, respectively, and adriamycin was the next most commonly used drug in $25 \%$ and $26.3 \%$, respectively. In the MEC group, most patients $(93.1 \%)$ were treated with oxaliplatin, and $28.2 \%-29.6 \%$ of patients were treated with 5-fluorouracil or capecitabine. These drugs were administered in combination. 
Table 1. Distribution of evaluable patients receiving cycles 1 to 3 of highly emetic or moderately emetic chemotherapy across countries

\begin{tabular}{lccrr} 
& & & \multicolumn{3}{c}{ Evaluable patients (HEC/MEC) } \\
\cline { 3 - 5 } Country & & Cycle 1 & Cycle 2 & Cycle 3 \\
Australia & 5 & 74 & 64 & 59 \\
China & 6 & 153 & 137 & 104 \\
India & 6 & 88 & 84 & 76 \\
South Korea & 6 & $80 / 71$ & $74 / 63$ & $68 / 56$ \\
Singapore & 2 & 57 & 53 & 49 \\
Taiwan & 6 & 125 & 123 & 121 \\
\hline
\end{tabular}

HEC, highly emetogenic chemotherapy; MEC, moderately emetogenic chemotherapy.

Table 2. Baseline characteristics of chemotherapy regimen used

\begin{tabular}{lcc} 
Regimen & South Korea $(\mathbf{n}=\mathbf{1 5 1})$ & AP total (n=648) \\
HEC regimen & & $145(45.6)$ \\
Breast & $19(23.8)$ & $90(28.3)$ \\
Lung & $24(30.0)$ & $30(9.4)$ \\
Other & $15(18.8)$ & $16(5.0)$ \\
Stomach & $12(15.0)$ & $93(29.2)$ \\
Metastatic disease (yes) & $37(46.3)$ & $127(38.5)$ \\
MEC regimen & & $54(16.4)$ \\
Colon & $43(60.6)$ & $44(13.3)$ \\
Lung & $2(2.8)$ & $30(9.1)$ \\
Other & $15(21.1)$ & $22(6.7)$ \\
Breast & 0 & $16(4.8)$ \\
Ovarian & $2(2.8)$ & $12(3.6)$ \\
Stomach & $7(9.9)$ & $11(3.3)$ \\
\hline Lymphoma & 0 & $157(47.6)$ \\
\hline Endometrial & 0 & $5(76.1)$ \\
\hline Metastatic disease (yes) & \\
\hline
\end{tabular}

Values are presented as number (\%). AP, Asia-Pacific; HEC, highly emetogenic chemotherapy; MEC, moderately emetogenic chemotherapy.

\section{Pattern of prescription in South Korea for CINV prophy- laxis in cycle 1}

\section{1) Antiemetic prophylaxis for HEC cycle 1}

The majority of patients $(46 / 80,57.5 \%)$ were initiated on a triple regimen, a combination of a corticosteroid, a 5 $\mathrm{HT}_{3}-\mathrm{RA}$, and an $\mathrm{NK}_{1}-\mathrm{RA}$, in the acute phase of the HEC regimen in South Korea compared with $123 / 318$ patients $(38.7 \%)$ in the AP region. A greater majority of patients (59/80, 73.8\%) were initiated on a double regimen (combination of a corticosteroid and an $\mathrm{NK}_{1}-\mathrm{RA}$ ) in the delayed phase of the HEC regimen in South Korea compared with $99 / 318$ (31.1\%) in the
AP region. All patients in South Korea were prescribed a primary antiemetic in the acute phase, but one patient in the AP region was not prescribed any antiemetic. However, in the delayed phase, $5 / 80$ patients $(6.3 \%)$ were not prescribed a primary antiemetic versus $75 / 318(23.6 \%)$ in the AP region. Table 3 summarizes the antiemetics prescribed for CINV prophylaxis during the acute and delayed phases of HEC cycle 1 according to antiemetic category for South Korea and the AP region.

\section{2) Antiemetic prophylaxis for MEC cycle 1}

The proportion of patients who were initiated on a double 
Table 3. Primary chemotherapy-induced nausea and vomiting prophylaxis for cycle 1 in the HEC and MEC

\begin{tabular}{|c|c|c|}
\hline Regimen & South Korea & AP total \\
\hline HEC regimen & 80 & 318 \\
\hline \multicolumn{3}{|l|}{ Acute phase } \\
\hline Corticosteroid alone & $1(1.3)$ & $5(1.6)$ \\
\hline 5HT3-RA alone & $2(2.5)$ & $28(8.8)$ \\
\hline $\mathrm{NK}_{1}-\mathrm{RA}$ alone & 0 & $2(0.6)$ \\
\hline Corticosteroid-5 $\mathrm{HT}_{3}-\mathrm{RA}$ & $24(30.0)$ & $146(45.9)$ \\
\hline Corticosteroid-NK1-RA & 0 & $4(1.3)$ \\
\hline $5 \mathrm{HT}_{3}-\mathrm{RA}-\mathrm{NK}_{1}-\mathrm{RA}$ & $7(8.8)$ & $9(2.8)$ \\
\hline Corticosteroid-5HT $3-\mathrm{RA}-\mathrm{NK}_{1}-\mathrm{RA}$ & $46(57.5)$ & $123(38.7)$ \\
\hline No primary antiemetic & 0 & $1(0.3)$ \\
\hline \multicolumn{3}{|l|}{ Delayed phase } \\
\hline Corticosteroid alone & $1(1.3)$ & $32(10.1)$ \\
\hline 5HT3-RA alone & $6(7.5)$ & $35(11.0)$ \\
\hline $\mathrm{NK}_{1}$-RA alone & $7(8.8)$ & $43(13.5)$ \\
\hline Corticosteroid-5 $\mathrm{HT}_{3}-\mathrm{RA}$ & $1(1.3)$ & $30(9.4)$ \\
\hline Corticosteroid-NK1-RA & $59(73.8)$ & $99(31.1)$ \\
\hline $5 \mathrm{HT}_{3}-\mathrm{RA}-\mathrm{NK}_{1}-\mathrm{RA}$ & $1(1.3)$ & $1(0.3)$ \\
\hline Corticosteroid-5HT $3-\mathrm{RA}-\mathrm{NK}_{1}-\mathrm{RA}$ & 0 & $3(0.9)$ \\
\hline No primary antiemetic & $5(6.3)$ & $75(23.6)$ \\
\hline MEC regimen & 71 & 330 \\
\hline \multicolumn{3}{|l|}{ Acute phase } \\
\hline Corticosteroid alone & $1(1.4)$ & $5(1.5)$ \\
\hline $5 \mathrm{HT}_{3}-\mathrm{RA}$ alone & $9(12.7)$ & $43(13.0)$ \\
\hline $\mathrm{NK}_{1}-\mathrm{RA}$ alone & 0 & $1(0.3)$ \\
\hline Corticosteroid-5HT3-RA & $59(83.1)$ & $260(78.8)$ \\
\hline Corticosteroid-5 $\mathrm{HT}_{3}-\mathrm{RA}-\mathrm{NK}_{1}-\mathrm{RA}$ & $2(2.8)$ & $17(5.2)$ \\
\hline No primary antiemetic & 0 & $4(1.2)$ \\
\hline \multicolumn{3}{|l|}{ Delayed phase } \\
\hline Corticosteroid alone & $9(12.7)$ & $54(16.4)$ \\
\hline $5 \mathrm{HT}_{3}-\mathrm{RA}$ alone & $23(32.4)$ & $74(22.4)$ \\
\hline $\mathrm{NK}_{1}$-RA alone & 0 & $4(1.2)$ \\
\hline Corticosteroid-5 $\mathrm{HT}_{3}-\mathrm{RA}$ & $23(32.4)$ & $61(18.5)$ \\
\hline Corticosteroid-NK $1-\mathrm{RA}$ & $3(4.2)$ & $7(2.1)$ \\
\hline Corticosteroid-5HT $3-\mathrm{RA}-\mathrm{NK}_{1}-\mathrm{RA}$ & 0 & $1(0.3)$ \\
\hline No primary antiemetic & $13(18.3)$ & $129(39.1)$ \\
\hline
\end{tabular}

Values are presented as number (\%). HEC, highly emetogenic chemotherapy; MEC, moderately emetogenic chemotherapy; $\mathrm{AP}$, Asia-Pacific; 5HT3-RA, 5-hydroxytryptamine-3 receptor antagonist; $\mathrm{NK}_{1}-\mathrm{RA}$, neurokinin-1 receptor antagonist.

regimen (combination of a corticosteroid and a $5 \mathrm{HT}_{3}-\mathrm{RA}$ ) in the acute phase of the MEC regimen was greater in South Korea than in the AP region (59/71 [83.1\%] vs. 260/330 [78.8\%]). For the delayed phase, a similar number of patients were initiated on $5 \mathrm{HT}_{3}$ - $\mathrm{RA}(23 / 71$ [32.4\%]) or the double regimen (23/71 [32.4\%]) in South Korea, which was slightly higher compared with patients in the AP region (74/330 [22.4\%] and 61/330 [18.5\%], respectively). A primary antiemetic was prescribed to all patients in South Korea in the acute phase, but $4 / 330$ patients $(1.2 \%)$ were not pre- scribed antiemetics in the AP region. However, in the delayed phase, $13 / 71$ patients $(18.3 \%)$ were not prescribed a primary antiemetic versus $129 / 330$ patients $(39.1 \%)$ in the AP region.

\section{ASCO QOPI results in cycle 1 and adherence to CINV prophylaxis guidelines}

In South Korea and the AP region, use of a $5 \mathrm{HT}_{3}-\mathrm{RA}$ was frequent in the acute phase for both HEC and MEC regimens; 
Table 4. Summary of the American Society of Clinical Oncology QOPI measures in cycle 1

\begin{tabular}{lcc} 
Patient & South Korea & AP total \\
HEC evaluable patients & 80 & 318 \\
QOPI-1 & $79(98.8)$ & $306(96.2)$ \\
QOPI-2 & $70(87.5)$ & $269(84.6)$ \\
QOPI-3 & $53(66.3)$ & $138(43.4)$ \\
QOPI-4 & $46(57.5)$ & $123(38.7)$ \\
MEC evaluable patients & 71 & 330 \\
QOPI-1 & $70(98.6)$ & $320(97.0)$ \\
QOPI-2 & $61(85.9)$ & $277(83.9)$ \\
\hline
\end{tabular}

Values are presented as number (\%). QOPI, Quality Oncology Practice Initiative; AP, Asia-Pacific; HEC, highly emetogenic chemotherapy; QOPI-1, 5-hydroxy tryptamine-3 receptor antagonist $\left(5 \mathrm{HT}_{3}-\mathrm{RA}\right)$ prophylaxis prescribed or administered with HEC and moderately emetogenic chemotherapy (MEC) (corresponds to QOPI Symptom Module \#26); QOPI-2, corticosteroid and $5 \mathrm{HT}_{3}-\mathrm{RA}$ prophylaxis prescribed or administered with HEC and MEC (corresponds to QOPI Symptom Module \#27); QOPI-3, aprepitant prescribed with HEC (corresponds to QOPI Symptom Module \#28); QOPI-4, antiemetic therapies appropriately prescribed for HEC and MEC; thus, for HEC, yes to QOPI-2 and QOPI-3, and for MEC, yes to QOPI-2 (corresponds to QOPI Symptom Module \#29).

therefore, patients meeting QOPI-1 exceeded 95\%. In South Korea, the triple regimen (corticosteroid- $5 \mathrm{HT}_{3}-\mathrm{RA}-\mathrm{NK}_{1}-\mathrm{RA}$ ) was initiated in $46 / 80$ patients $(57.5 \%$ ) for acute management of CINV in cycle 1 of the HEC regimen, and the proportion of patients meeting QOPI-3 was $66.3 \%$. In the MEC regimen, the double regimen (corticosteroid-5 $5 \mathrm{HT}_{3}-\mathrm{RA}$ ) was initiated in 59/71 (83.1\%) for control of acute CINV in cycle 1 and $61 / 71(85.9 \%)$ patients in Korea met QOPI-2. In the HEC regimen, $46 / 80$ patients $(57.5 \%)$ in South Korea met QOPI-4. These measurements were higher than those in the AP regional data, in which a greater mismatch in guideline adherence was reported (HEC group [corticosteroid-5 $\mathrm{HT}_{3}-$ RA-NK 1 -RA], 38.7\% vs QOPI-3, 43.4\%; MEC group [corticosteroid-5 $\mathrm{HT}_{3}-\mathrm{RA}$ ], $78.8 \%$ vs. QOPI-2, 83.9\%) in acute phase of cycle 1 in the HEC regimen, respectively (Table 4).

The survey showed that the National Comprehensive Cancer Network (NCCN) guidelines were the antiemesis guidelines most commonly cited by physicians in Korea and across the AP region (except in Australia, where the MASCC/ European Society for Medical Oncology guidelines [8] were most commonly cited).

\section{Discussion}

We analyzed the pattern of antiemetic therapy in countries of the AP region in a previous report [15]. In that data, we compared the different patterns among countries associated with different policy, standard of care, or preference. In the current study, we tried to focus on the characteristics of the antiemetic therapy pattern in Korea, because knowledge regarding the different treatment patterns in real practice is important when establishing the principle for cancer treatment. The results showed that in accordance with guideline recommendations $[6,7]$, the majority of patients $(57.5 \%)$ received prophylaxis with a triple regimen (corticosteroid$5 \mathrm{HT}_{3}-\mathrm{RA}-\mathrm{NK}_{1}-\mathrm{RA}$ ) in the acute phase of cycle 1 in the HEC regimen in South Korea. A greater majority $(73.8 \%)$ of patients received prophylaxis with corticosteroid-NK 1 -RA in the delayed phase of cycle 1 in the HEC regimen. In the case of MEC in South Korea, a double regimen (corticosteroid-5 $5 \mathrm{HT}_{3}$-RA [83.1\%]) was initiated in a majority of patients in the acute phase of cycle 1. Interestingly, an equal proportion of patients were initiated on either a $5 \mathrm{HT}_{3}-\mathrm{RA}$ or a corticosteroid-5 $\mathrm{HT}_{3}-\mathrm{RA}$ (32.4\%) regimen in the delayed phase. NK $\mathrm{NK}_{1}$-RA prescriptions were noted in $66.3 \%$ and $83.8 \%$ of patients in the acute and delayed phases of HEC cycle 1, respectively, although $\mathrm{NK}_{1}-\mathrm{RA}$ (aprepitant) prescriptions as part of the HEC regimen for CINV prophylaxis are eligible for reimbursement in Korea. This is because of the reimbursement policy. Because this study was an observational study and no intervention was added to the treatment, it can reflect the treatment pattern in real practice. Many clinicians tend to choose treatment according to reimbursement guidelines in practice, but it can be interpreted narrowly according to type of drug, duration, frequency of symptoms, timing of initiation, and so on. This could lead to the limited prescription of anti-emetics in the acute phase of cycle 1 chemotherapy, which has limited application in real practice. Considering that many clinicians tend to choose the drug based 
on the reimbursement policy, it has to be changed flexible for better and more comfortable treatment of patients.

Therefore, considering international guideline recommendations [6,7], there is room for improvement in $\mathrm{NK}_{1}-\mathrm{RA}$ based triple regimen prophylaxis in the HEC groups. This gap was even more evident for CINV prophylaxis in the acute phase and delayed phase of cycle 1 in both HEC and MEC groups. For better control of CINV prophylaxis in the acute phase of cycle 1 chemotherapy, we suggested that reimbursement policy should be applied flexibly, not restricted to reimbursement guidelines. However, CINV prophylaxis in Korea could not be directly compared with that of the AP region due to large variations in prescribing patterns for CINV prophylaxis among countries.

The differences between South Korea and the AP region could be attributed to country-specific differences in factors such as adherence to guidelines, dosage and prescribing patterns of CINV prophylaxis therapy [1,10-13,15], prevalence of quality-of-care indicators [15], use of rescue medication, drug availability, reimbursement policies, location, and study design [19-21].

In this study, the NCCN guidelines [7] were the most commonly cited guidelines in the AP region, including South Korea. In the MEC regimen, the double regimen (corticosteroid-5 $\mathrm{HT}_{3}-\mathrm{RA}$ ) was initiated in $59 / 71$ patients $(83.1 \%)$ for control of acute CINV in cycle 1 and $61 / 71$ patients $(85.9 \%)$ met the QOPI-2 rate in South Korea. The triple regimen (corticosteroid-5 $\mathrm{HT}_{3}-\mathrm{RA}-\mathrm{NK}_{1}-\mathrm{RA}$ ) was initiated in $46 / 80$ patients $(57.5 \%)$ for management of acute CINV in cycle 1 of the HEC regimen, and $66.3 \%$ of patients met QOPI-3. These rates were higher than those reported for the AP region, where a higher mismatch in guideline adherence was reported. Considering the discrepancy between antiemetic prophylaxis and the actual requirement based on qualityof-care indicators (ASCO QOPI), adherence to international guidelines for CINV prophylaxis must improve, particularly in the acute phase of cycle 1 in HEC in South Korea and more so in the AP region.

As mentioned in previous publications [14-18], the PrACTICE study had several limitations: (1) the sample size in 3 countries (Australia, India, and Singapore) did not reach the planned enrollment of 45 patients in each of the HEC and MEC cohorts for comparison with the South Korean population; (2) CINV findings were patient reported and based on patient diary completion of the MAT on postchemotherapy days 2 and 6. However, sites that reviewed diary entries confirmed that the recorded information included the patient's verbal description of their experiences; (3) QOPI criteria do not take into account the prescription schedule on the appropriate day [25]; for example, QOPI-2 criterion includes any corticosteroid (and $5 \mathrm{HT}_{3}-\mathrm{RA}$ ) prophylaxis initiated with HEC, and not a specific corticosteroid, on days 1-4 in HEC; and (4) the availability of antiemetic medications and reimbursement differ in each country, which may likely influence the antiemetic prescription patterns.

\section{Conclusion}

Despite relatively higher rates than the AP region, management of CINV in cycle 1 of HEC in Korea should be more active.

\section{Conflicts of Interest}

This study was funded by Merck \& Co., Inc. Medical writing services were provided by Dr. Annirudha Chillar of Cactus Communications and funded by Merck \& Co., Inc. The authors retained full control of the manuscript content.

\section{References}

1. Jordan K, Gralla R, Jahn F, Molassiotis A. International antiemetic guidelines on chemotherapy induced nausea and vomiting (CINV): content and implementation in daily routine practice. Eur J Pharmacol. 2014;722:197-202.

2. Bloechl-Daum B, Deuson RR, Mavros P, Hansen M, Herrstedt J. Delayed nausea and vomiting continue to reduce patients' quality of life after highly and moderately emetogenic chemotherapy despite antiemetic treatment. J Clin Oncol. 2006; 24:4472-8.

3. Hassan BA, Yusoff ZB. Genetic polymorphisms in the three malaysian races effect granisetron clinical antiemetic actions in breast cancer patients receiving chemotherapy. Asian Pac J Cancer Prev. 2011;12:185-91.

4. Molassiotis A, Aapro M, Dicato M, Gascon P, Novoa SA, Isambert $\mathrm{N}$, et al. Evaluation of risk factors predicting chemotherapy-related nausea and vomiting: results from a European prospective observational study. J Pain Symptom Manage. 2014;47:839-48.e4.

5. Pirri C, Katris P, Trotter J, Bayliss E, Bennett R, Drummond P. Risk factors at pretreatment predicting treatment-induced 
nausea and vomiting in Australian cancer patients: a prospective, longitudinal, observational study. Support Care Cancer. 2011;19:1549-63.

6. Basch E, Hesketh PJ, Kris MG, Prestrud AA, Temin S, Lyman GH. Antiemetics: American Society of Clinical Oncology clinical practice guideline update. J Oncol Pract. 2011;7:395-8.

7. National Comprehensive Cancer Network. Antiemesis [Internet]. Fort Washington, PA: National Comprehensive Cancer Network; 2014 [cited 2014 Dec 26]. Available from: http:// www.nccn.org.

8. Roila F, Herrstedt J, Aapro M, Gralla RJ, Einhorn LH, Ballatori E, et al. Guideline update for MASCC and ESMO in the prevention of chemotherapy- and radiotherapy-induced nausea and vomiting: results of the Perugia consensus conference. Ann Oncol. 2010;21 Suppl 5:v232-43.

9. Herrstedt J, Roila F; ESMO Guidelines Working Group. Chemotherapy-induced nausea and vomiting: ESMO clinical recommendations for prophylaxis. Ann Oncol. 2008;19 Suppl 2:ii110-2.

10. Caracuel F, Munoz N, Banos U, Ramirez G. Adherence to antiemetic guidelines and control of chemotherapy-induced nausea and vomiting (CINV) in a large hospital. J Oncol Pharm Pract. 2015;21:163-9.

11. Affronti ML, Schneider SM, Herndon JE 2nd, Schlundt S, Friedman HS. Adherence to antiemetic guidelines in patients with malignant glioma: a quality improvement project to translate evidence into practice. Support Care Cancer. 2014; 22:1897-905.

12. Fujii $H$, Iihara $H$, Ishihara $M$, Takahashi $T$, Yoshida $K$, Itoh $Y$. Improvement of adherence to guidelines for antiemetic medication enhances emetic control in patients with colorectal cancer receiving chemotherapy of moderate emetic risk. Anticancer Res. 2013;33:5549-56.

13. Gilmore JW, Peacock NW, Gu A, Szabo S, Rammage M, Sharpe J, et al. Antiemetic guideline consistency and incidence of chemotherapy-induced nausea and vomiting in US community oncology practice: INSPIRE Study. J Oncol Pract. 2014; 10:68-74.

14. Hsieh RK, Chan A, Kim HK, Yu S, Kim JG, Lee MA, et al. Baseline patient characteristics, incidence of CINV, and physician perception of CINV incidence following moderately and highly emetogenic chemotherapy in Asia Pacific countries. Support Care Cancer. 2015;23:263-72.

15. Yu S, Burke TA, Chan A, Kim HK, Hsieh RK, Hu X, et al. Antiemetic therapy in Asia Pacific countries for patients receiving moderately and highly emetogenic chemotherapy: a descriptive analysis of practice patterns, antiemetic quality of care, and use of antiemetic guidelines. Support Care Cancer. 2015;23:273-82.

16. Kim HK, Hsieh R, Chan A, Yu S, Han B, Gao Y, et al. Impact of CINV in earlier cycles on CINV and chemotherapy regimen modification in subsequent cycles in Asia Pacific clinical practice. Support Care Cancer. 2015;23:293-300.

17. Chan A, Kim HK, Hsieh RK, Yu S, de Lima Lopes G Jr, Su WC, et al. Incidence and predictors of anticipatory nausea and vomiting in Asia Pacific clinical practice: a longitudinal analysis. Support Care Cancer. 2015;23:283-91.

18. Keefe DM, Chan A, Kim HK, Hsieh RK, Yu S, Wang Y, et al. Rationale and design of the Pan Australasian chemotherapyinduced emesis burden of illness study. Support Care Cancer. 2015;23:253-61.

19. Liau CT, Chu NM, Liu HE, Deuson R, Lien J, Chen JS. Incidence of chemotherapy-induced nausea and vomiting in Taiwan: physicians' and nurses' estimation vs. patients' reported outcomes. Support Care Cancer. 2005;13:277-86.

20. Chan A, Shih V, Chew L. Evolving roles of oncology pharmacists in Singapore: a survey on prescribing patterns of antiemetics for chemotherapy induced nausea and vomiting (CINV) at a cancer centre. J Oncol Pharm Pract. 2008;14:23-9.

21. So WK, Chan DN, Chan HY, Krishnasamy M, Chan T, Ling WM, et al. Knowledge and practice among Hong Kong oncology nurses in the management of chemotherapy-induced nausea and vomiting. Eur J Oncol Nurs. 2013;17:370-4.

22. Hesketh PJ, Kris MG, Grunberg SM, Beck T, Hainsworth JD, Harker G, et al. Proposal for classifying the acute emetogenicity of cancer chemotherapy. J Clin Oncol. 1997;15:103-9.

23. Molassiotis A, Coventry PA, Stricker CT, Clements C, Eaby B, Velders L, et al. Validation and psychometric assessment of a short clinical scale to measure chemotherapy-induced nausea and vomiting: the MASCC antiemesis tool. J Pain Symptom Manage. 2007;34:148-59.

24. Uppsala Monitoring Center. 2010 WHO Drug Dictionary version 3 [Internet]. Uppsala: Uppsala Monitoring Center; 2010 [cited 2014 Dec 26]. Available from: http://www.umc-products.com.

25. American Society of Clinical Oncology. The Quality Oncology Practice Initiative (QOPI) and the QOPI Certification Program [Internet]. Alexandria, VA: American Society of Clinical Oncology; 2014 [cited 2014 Dec 26]. Available from: http:// qopi.asco.org/. 